\title{
A novel mono-modality fusion imaging method based on three-dimensional contrast-enhanced ultrasound for the evaluation of ablation margins after microwave ablation of hepatocellular carcinoma
}

\author{
Jianmin Ding ${ }^{1,2,3 \#}$, Dong Wang ${ }^{1,3}$, Yan Zhou ${ }^{1,3}$, Lin Zhao ${ }^{1,3}$, Hongyu Zhou ${ }^{1,3}$, Xiang Jing ${ }^{1,3}$, \\ Yandong Wang ${ }^{1,3}$ \\ ${ }^{1}$ Department of Ultrasound, Tianjin Third Central Hospital, Tianjin, China; ${ }^{2}$ The Third Central Clinical College of Tianjin Medical University, \\ Tianjin, China; ${ }^{3}$ Tianjin Institute of Hepatobiliary Disease, Tianjin Key Laboratory of Extracorporeal Life Support for Critical Diseases, Artificial \\ Cell Engineering Technology Research Center, Tianjin Third Central Hospital, Tianjin, China \\ Contributions: (I) Conception and design: X Jing, J Ding, D Wang; (II) Administrative support: X Jing; (III) Provision of study materials or patients: X \\ Jing, J Ding, Y Wang; (IV) Collection and assembly of data: J Ding, Y Zhou; (V) Data analysis and interpretation: J Ding, Y Zhou; (VI) Manuscript \\ writing: All authors; (VII) Final approval of manuscript: All authors. \\ \#These authors contributed equally to this work. \\ Correspondence to: Xiang Jing. Tianjin Third Central Hospital, Tianjin 300170, China. Email: dr.jingxiang@aliyun.com.
}

Background: To investigate the feasibility and accuracy of using preoperative and postoperative threedimensional contrast-enhanced ultrasound (3D CEUS) fusion imaging in the evaluation of safety margins after thermal ablation of hepatocellular carcinoma (HCC).

Methods: A total of the 24 patients with HCC who underwent microwave ablation (MWA) between June 2020 and December 2020 were enrolled in this study. All patients received preoperative and postoperative $3 \mathrm{D}$ CEUS. The preoperative and postoperative 3D CEUS images were then fused. The success rate and evaluation time were recorded. The ablation margin and whether or not the safety margin was reached were calculated and recorded. If the ablation safety margin was not reached, the residual ablation volume needed to cover the safety margin was calculated automatically. The agreement between contrast-enhanced computed tomography(CECT) and 3D CEUS fusion imaging in the evaluation of ablation margins was explored using the kappa coefficient.

Results: The 3D CEUS fusion success rate was $95.8 \%(23 / 24)$, with a mean fusion time of $(4.1 \pm 1.8)$ minutes. Twenty-three tumors were completely ablated, and the safety margin was achieved for 9 tumors. The ablation margin of 14 tumors was $<5 \mathrm{~mm}$. The mean uncovered safety margin volume was $(2.27 \pm 2.11) \mathrm{mL}$, and the mean proportion of the uncovered safety margin to the whole safety margin was $16.8 \%$. According to the results of preoperative and postoperative CECT fusion imaging, the ablation margin of 13 tumors was $<5 \mathrm{~mm}$, and the ablation margin of 10 tumors was $>5 \mathrm{~mm}$. The 2 methods showed excellent consistency, with a Kappa value of $0.911(\mathrm{P}=0.000012)$.

Conclusions: This study has presented a novel mono-modality fusion imaging method based on CEUS. We demonstrated that 3D CEUS fusion has a short fusion time and a high success rate, as well as good consistency with enhanced CT fusion. Therefore, 3D CEUS fusion is a feasible and accurate tool for evaluating the immediate efficacy of thermal ablation of HCC.

Keywords: Three-dimensional contrast-enhanced ultrasound fusion imaging (3D CEUS fusion imaging); ablation margin; hepatocellular carcinoma (HCC); thermal ablation

Submitted Jan 14, 2021. Accepted for publication Feb 08, 2021.

doi: 10.21037/jgo-21-46

View this article at: http://dx.doi.org/10.21037/jgo-21-46 


\section{Introduction}

Image-guided local thermal ablation, including radiofrequency ablation and microwave ablation (MWA), represents the third radical treatment for hepatocellular carcinoma (HCC) besides liver transplantation and surgical resection $(1,2)$. For patients with early HCC, thermal ablation therapy and surgical resection can achieve similar overall survival, but local progression-free survival is better with surgical resection than with thermal ablation therapy $(3,4)$. Several previous studies have demonstrated that an insufficient ablation margin is an important risk factor for local tumor progression after thermal ablation treatment (5-7). Therefore, accurate assessment of the ablation margin following thermal ablation is crucial. Consequently, the safety margin for thermal ablation has always been a hot topic in the field of HCC research.

In previous studies, contrast-enhanced ultrasound (CEUS) and contrast enhanced computed tomography (CECT) or contrast enhanced magnetic resonance imaging (CEMRI) were used for side-by-side evaluation of ablation margins $(8,9)$; however, these methods are subjective and cannot be used for accurate assessment. Thermal ablation margins have been evaluated using fusion imaging navigation systems, which fuse the pre-ablation image with the post-ablation image (10).

Some for the previous studies used pre- and postablation CT/MRI-CT/MRI and CT/MRI-2DCEUS as the reference standard evolution methods for ablation margin and obtained satisfactory results $(11,12)$. However, several limitations hinder the application of the aforementioned methods in clinical practice. First, a repeated CT or MRI scan, which is not recommended by the guidelines, is required for evaluation of the ablation margin within 24 hours or 1 week after thermal ablation; however, this increases the patient's medical costs and radiation exposure. Second, it is difficult to obtain CT and MRI data in most centers. Third, CT and MRI data are not convenient for immediate evaluation as in most cases thermal ablations are guided by ultrasound rather than CT or MRI. Therefore, a mono-modality fusion imaging method that can be used for more convenient and timely evaluation of the ablation margin is needed.

As a real-time, repeatable, and non-radioactive imaging method, ultrasound is used for thermal ablation therapy. A mono-modality fusion imaging method for ablation margin evaluation was developed by Minami et al. based on US and CEUS (13). In most studies 3D US/CEUS is fused with $2 \mathrm{D}$ real-time US/CEUS to evaluate the ablation margin.
However, 2D real-time US/CEUS is dependent on the experience of the operators and might lead to the inevitable omission of information on the other 2 planes $(14,15)$. Due to its higher spatial resolution, 3D CEUS can obtain more information than 2D CEUS and has been used in the immediate evaluation of thermal ablation margins in the treatment of HCC (16). Therefore, 3D CEUS-3D CEUS fusion imaging may be the optimal method for evaluating ablation margins, with the advantages of being convenient and timely. Ye et al. (17) also attempted to evaluate ablation margins using 3D CEUS- 3D CEUS fusion imaging and presented some primary results demonstrating that this method is available. However, further improvements are still needed, including in the aspects of the evaluation algorithm and image visualization.

Here, we present a novel method for evaluation of the margin of ablation based on the 3D CEUS fusion imaging of pre- and post-thermal ablation. In the present study, we explored the feasibility and accuracy of 3D CEUS fusion imaging and compared its results with those of enhanced CT fusion imaging for the evaluation of the ablation margin after thermal ablation treatment. We present the following article in accordance with the MDAR checklist (available at http://dx.doi.org/10.21037/jgo-21-46).

\section{Methods}

Approval for this prospective study was granted by the institutional review board of Tianjin Third Central Hospital, and informed consent was obtained from all patients. All procedures performed in this study involving human participants were in accordance with the Declaration of Helsinki (as revised in 2013). Twenty-four patients with HCC who received MWA in our center between June 2020 and December 2020 met the study inclusion criteria. The patients in this study were diagnosed with HCC according to the American Association for the Study of Liver Diseases guidelines for the treatment of HCC (18).

The inclusion criteria were as follows: (I) patient with age $>18$ and $<75$ years met the HCC diagnostic criteria; (II) patient met the Milan criteria, which are a single HCC measuring $\leq 5 \mathrm{~cm}$ or $\leq 3$ nodules measuring $<3 \mathrm{~cm}$ each; (III) liver dysfunction corresponding to Child-Pugh class A or B, with no ascites or only a small amount of ascites; (IV) tumor clearly visible on ultrasound, with safe puncture pathways; and (V) enhanced CT within the 2 weeks before the operation and within the 1 week after the operation

The exclusion criteria were as follows: (I) severe 


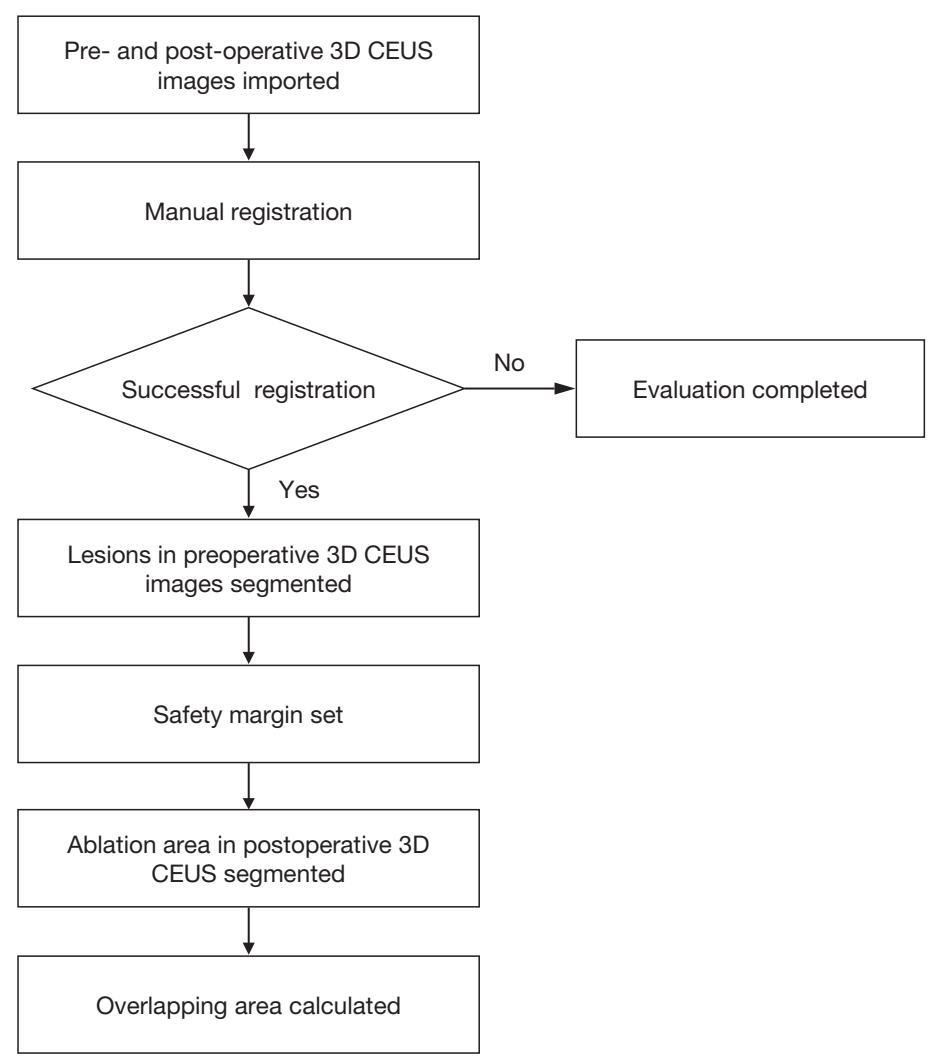

Figure 1 Flowchart for the evaluation of ablation results using 3D CEUS. 3D CEUS, three-dimensional contrast-enhanced ultrasound.

contraindications to thermal ablation therapy (such as cardiopulmonary, liver, kidney dysfunction, or coagulation disorders); (II) the tumor was adjacent to a previously ablated area; and (III) invasion of vessels or distant metastases.

\section{D CEUS examination}

US images were obtained with the Philips EPIQ 7 ultrasound system (Philips Medical System) equipped with a C5-1 (1.0-5.0 MHz) convex array probe, pulse inversion imaging (PI) software, and a mechanical index of 0.04-0.08. The PercuNav feature of the Philips system was used to acquire and reconstruct the $3 \mathrm{D}$ ultrasound volumes. Sulfur hexafluoride microbubble (SF6) contrast agent (SonoVue, Bracco, Milan, Italy) was mixed with $5 \mathrm{ml}$ saline before bolus injection into the antecubital vein. Then, 1.2 to $2.0 \mathrm{~mL}$ of contrast agent was intravenously injected via the antecubital vein, followed by flushing with $5 \mathrm{~mL} 0.9 \%$ sodium chloride solution. When the tumor was clearly visible in arterial phase, the clinician performed a freehand sweep to acquire a set of images depicting the tumor. These images were reconstructed into a pre-operative B-mode ultrasound volume, $V_{P r e B}$, and the corresponding $3 \mathrm{D}$ CEUS volume, $V_{\text {Pre_C }}$. These 2 volumes were used as a reference before ablation.

Approximately 10 to 15 minutes after the ablation, the clinician examined the tumor again using CEUS. When the ablated area was clearly visible in portal phase, the clinician performed a freehand sweep using the same acoustic window as in the previous sweep. The images collected in this sweep were reconstructed into a post-operative B-mode ultrasound volume, $V_{P_{\text {Ost_ } B},}$ and the corresponding $3 \mathrm{D}$ CEUS volume, $V_{\text {Post_C. }}$

To avoid distortion of the reconstructed $3 \mathrm{D}$ volumes caused by respiratory motion of the liver during acquisition, all the sweeps were performed at the end of the expiratory phase. All 3D CEUS examinations were performed by the same investigator with 15 years of ultrasound experience. If a patient had multiple lesions, only the largest lesion was included in the study.

Figure 1 shows the flowchart for the evaluation of 

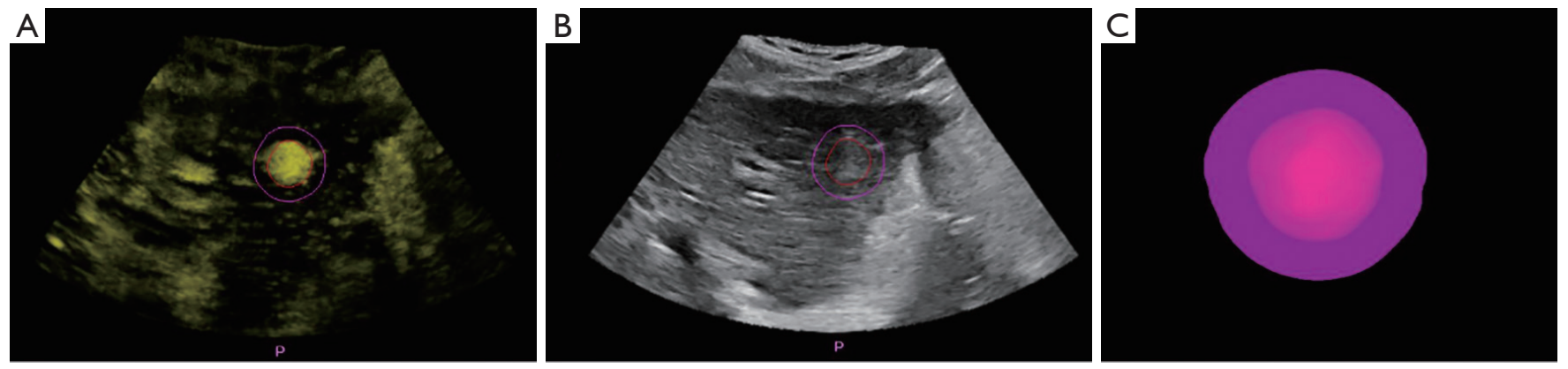

Figure 2 Illustration of 3D view of tumor with a semi-automatic 3D contouring method. (A) A plane slice of the preoperative 3D CEUS volume with the tumor clearly shown. The red line is the margin of tumor, and the purple line is the 5-mm safety margin. (B) A plane slice of the preoperative 3D US volume in the same position as (A). (C) A 3D view of the tumor with the safety margin. 3D CEUS, threedimensional contrast-enhanced ultrasound.

ablation results using 3D CEUS fusion imaging. The 3D US volumes were acquired using the research mode in PercuNav, which allowed the use of some unreleased function. The first step was to align the preoperative $3 \mathrm{D}$ CEUS volume with the postoperative 3D CEUS volume. Manual registration was performed based on common landmarks that were visible in both volumes. If landmarks could not be identified reliably, then the corresponding 3D B-mode US volumes would be used to aid the registration. The fusion was considered successful if the difference in the positions of an anatomical structure before and after therapy was $<3 \mathrm{~mm}$ after superimposition. The time taken for registration and the success rate of registration were recorded. Two operators (Jinmin Ding and Xiang Jing) performed and interpreted the 3D CEUS fusion imaging process together and consensus was reached through discussion.

After a successful registration, the operator then segmented the margins of the tumor and ablation area from the preoperative and postoperative 3D CEUS volumes, respectively, using a semi-automatic $3 \mathrm{D}$ contouring method with edge detection capability. The semi-automatic contouring algorithm could also automatically compute the safety margin to the $3 \mathrm{D}$ tumor contour specified by the operator. As shown in Figure 2, after complete segmentation of the tumor and ablation area, the $3 \mathrm{D}$ view of the tumor and safety margins could be observed.

The final step was to evaluate the ablation performance. In this study, the main evaluation criterion was the coverage of the safety margin by the ablation area. Using a research feature of PercuNav, the coverage of the tumor and its safety margin were computed from the $3 \mathrm{D}$ contour of the tumor and the ablation area. Then, the assessment results were divided into three categories: complete ablation of the tumor and its safety margin; complete ablation of the tumor but incomplete ablation of the safety margin; and incomplete ablation of the tumor (Figure 3). If the ablation area did not completely cover the safety margin, the volume of the uncovered safety margin and the proportion of the uncovered safety margin to the total safety margin were calculated and recorded.

It is worth noting that when the distance of lesion to a vessel or liver surface is $<5 \mathrm{~mm}$, the 5 -mm safety margin must be carefully defined to ensure that the safety margin will not cover the vessel or extend beyond the liver (Figure 4).

\section{Enhanced CT examination}

Dynamic CECT scanning was performed using the Somatom Definition Flash dual energy CT scanner as previous study mentioned (19). Iohexol (Beijing Beilu Pharmaceutical Co., Ltd., China), the contrast agent, at a dosage of $1.2 \mathrm{~mL} / \mathrm{kg}$ body weight and a flow rate of $3.5 \mathrm{~mL} / \mathrm{s}$, was injected via the median cubical vein. The acquisition of images in the hepatic arterial phase started with a delay of 25 seconds to 35 seconds after the threshold had been reached. The acquisition of portal venous phase images and late phase images started at about 50 to 70 seconds and 180 seconds after the initiation of contrast injection, respectively.

\section{Enhanced CT fusion and evaluation of the ablation margin}

For CT evaluation, the operator opened the PercuNav 

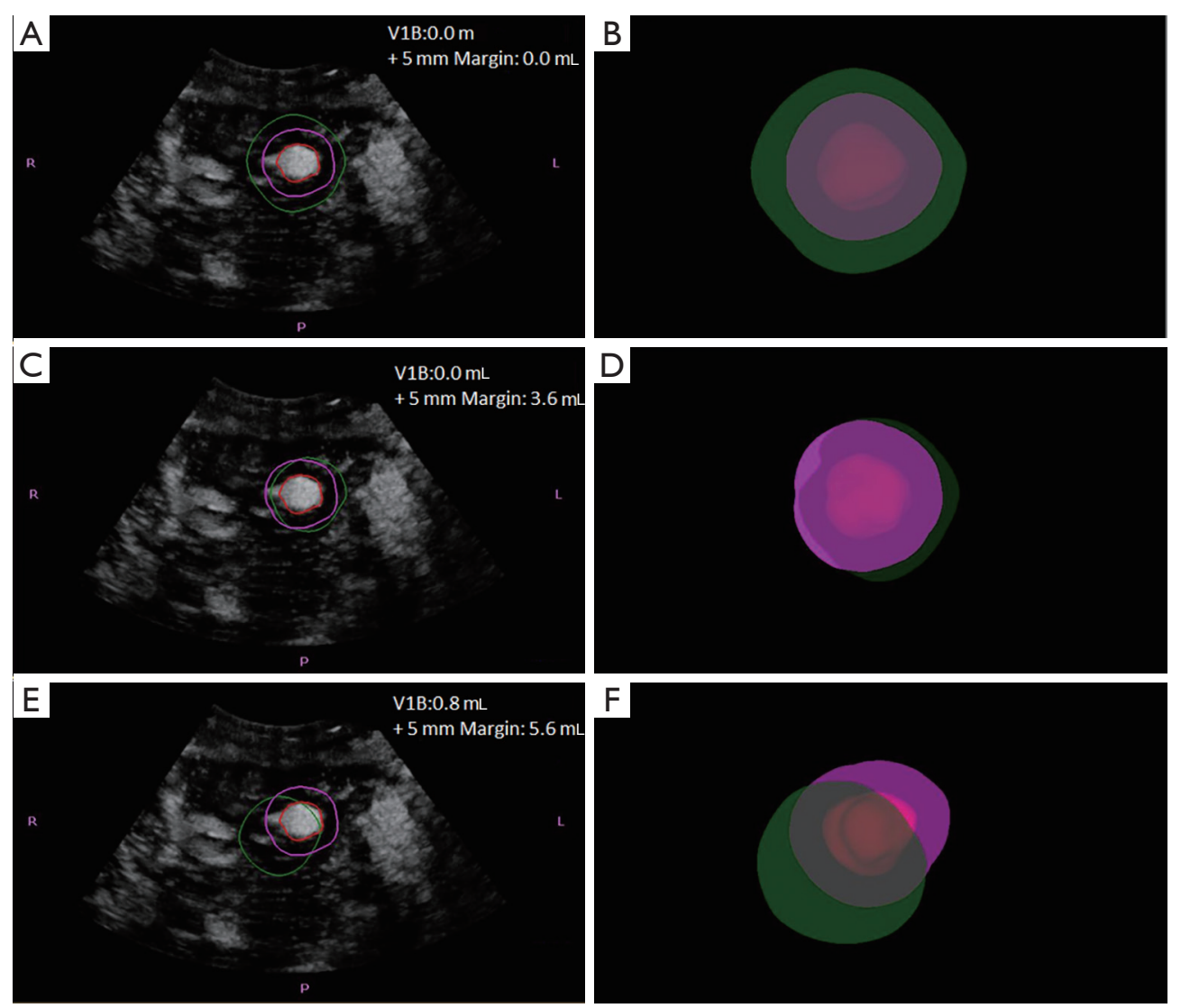

Figure 3 Illustration the assessment results of 3D CEUS fusion imaging. (A) Image of a case with complete ablation. The red line is the tumor margin, the purple line is the safety margin, and the green line is the ablation margin. V1B and +5 mm margin represent the residual volume of the tumor and the safety margin, respectively. (C) Image of a case with complete ablation of the tumor but not the safety margin. (E) Image of a case with incomplete ablation of both the tumor and the safety margin. (B,D,F) Show the 3D views of (A,C,E), respectively. 3D, three-dimensional.
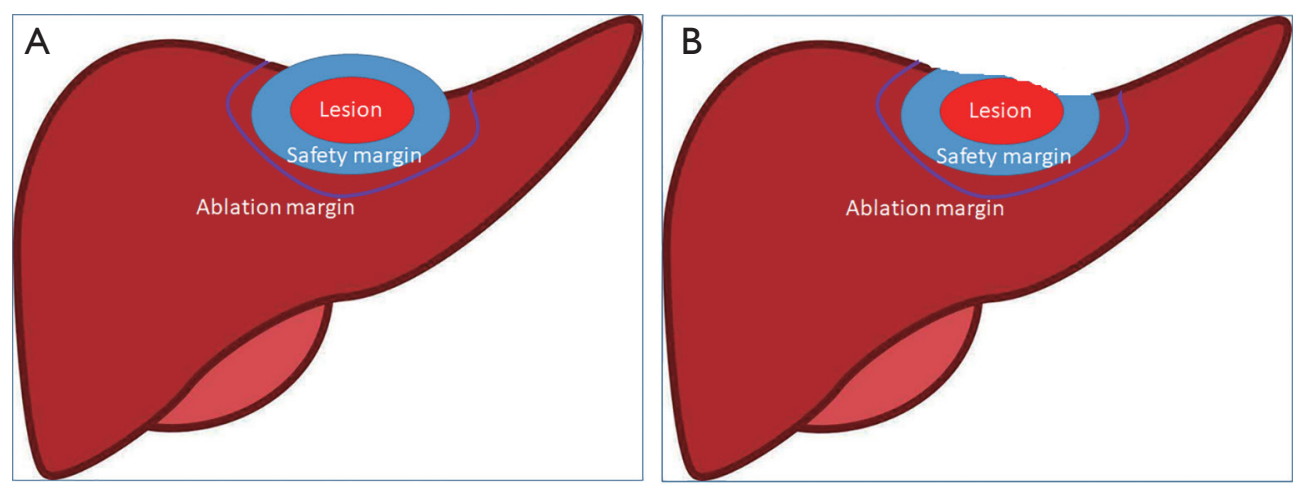

Figure 4 A schematic diagram of a case of a lesion close to the liver surface. The red region is the lesion, the blue region is the safety margin, and the purple line represents the ablation margin. (A) With the safety margin set as $5 \mathrm{~mm}$, the liver area will be extended, and the residual ablation volume will never be $0 \mathrm{~mL}$. (B) If the safety margin to extend the liver area is not considered, the residual ablation volume will be $0 \mathrm{~mL}$. 
Table 1 Clinical characteristics of 24 patients who received microwave ablation treatment for hepatocellular carcinoma

\begin{tabular}{lc}
\hline Characteristic & Value \\
\hline Patient $(\mathrm{n})$ & 24 \\
Mean age $(\mathrm{y})^{*}$ & $61.5 \pm 8.0$ \\
Sex & 18 \\
Male & 6 \\
Female & 23 \\
Known cirrhosis & \\
Etiology of liver disease & 20 \\
Hepatitis B virus & 2 \\
Hepatitis C virus & 1 \\
Alcohol & 1 \\
Autoimmune hepatitis & 0 \\
Other cause &
\end{tabular}

No. of tumors ( $n$ )

1

2

3

Tumor size $(\mathrm{cm})^{\star}$

Tumor location

Adjacent blood vessel $\quad 9$

Adjacent liver capsule

Adjacent is defined as $<5 \mathrm{~mm}$; blood vessel diameter $>3 \mathrm{~mm}$. *, data are mean \pm standard deviation.

software and chose CT/MR/PET model, which has a fusion function for importing preoperative and postoperative CT images. The CT evaluation process was the same as the CEUS evaluation process and comprised 3 parts: registration, segmentation, and evaluation. Patients' CT data were imported into the PercuNav system in the DICOM format via a USB flash drive. Registration with internal landmarks was used for image fusion. Three anatomical landmarks on the CT images before therapy and 3 at the corresponding positions on CT images after therapy were selected for registration. Fusion was considered successful if the difference between the positions of an anatomical structure before and after therapy was $<3 \mathrm{~mm}$ after superimposition. The CT fusion process was performed and interpreted by 2 operators mentioned above who were blinded to the $3 \mathrm{D}$ CEUS fusion results, and consensus was reached by discussion.

\section{Thermal ablation procedures}

MWA procedures were carried out using a MTC-3 microwave (MW) therapy instrument (Forsea Microwave \& Electronic Research Institute, Nanjing, China) with a frequency of 2,450 MHz and an output power of 50-100 W. The MW antenna was a $14 \mathrm{G}$ unipolar cooled-shaft needle measuring $15 \mathrm{~cm}$ in length with a 1.5 -cm active tip.

MWA was carried out under ultrasound guidance by an operator with 10 years of experience in ablation (D. J. M.). The MW radiator was inserted. The MWA output power was $60-80 \mathrm{~W}, 10$ minutes per point. If the tumor size was $\leq 1.5 \mathrm{~cm}$, single-needle ablation was performed. For tumors of $>1.5 \mathrm{~cm}$, a multi-needle overlapping ablation plan was made according to the shape, size, and location of the tumor. The ablation range exceeded the tumor margin by $0.5 \mathrm{~cm}$. All of the patients received CECT examination one month after thermal ablation to confirm the local treatment efficacy.

\section{Statistical analysis}

Data were expressed as the median \pm standard deviation (SD) (range). The agreement between CT and 3D CEUS fusion in the evaluation of the ablation margin was expressed by the kappa coefficient. All data were analyzed using SPSS version 22.0 software (SPSS, Chicago, IL, USA), and a P value of $<0.05$ was considered to be statistically significant.

\section{Results}

\section{Patient characteristics}

Thirty-two lesions of 24 patients received MWA treatment, of which 24 lesions received 3D CEUS fusion and enhanced CT fusion before and after treatment. Among the patients were 18 males and 6 females, ranging in age from 46.0 to $77.0(61.5 \pm 8.0)$ years old. The maximum lesion diameter was $(2.3 \pm 0.6)$ (range, $1.5-3.4) \mathrm{cm}$. The basic clinical characteristics of the patients are detailed in Table 1 .

\section{D CEUS fusion and evaluation of the ablation margin}

The success rate of 3D CEUS fusion of images before and after MWA treatment was $95.8 \%$ (23/24), and the fusion time was 2-9 (4.1 \pm 1.8$)$ minutes. Twenty-three tumors were 

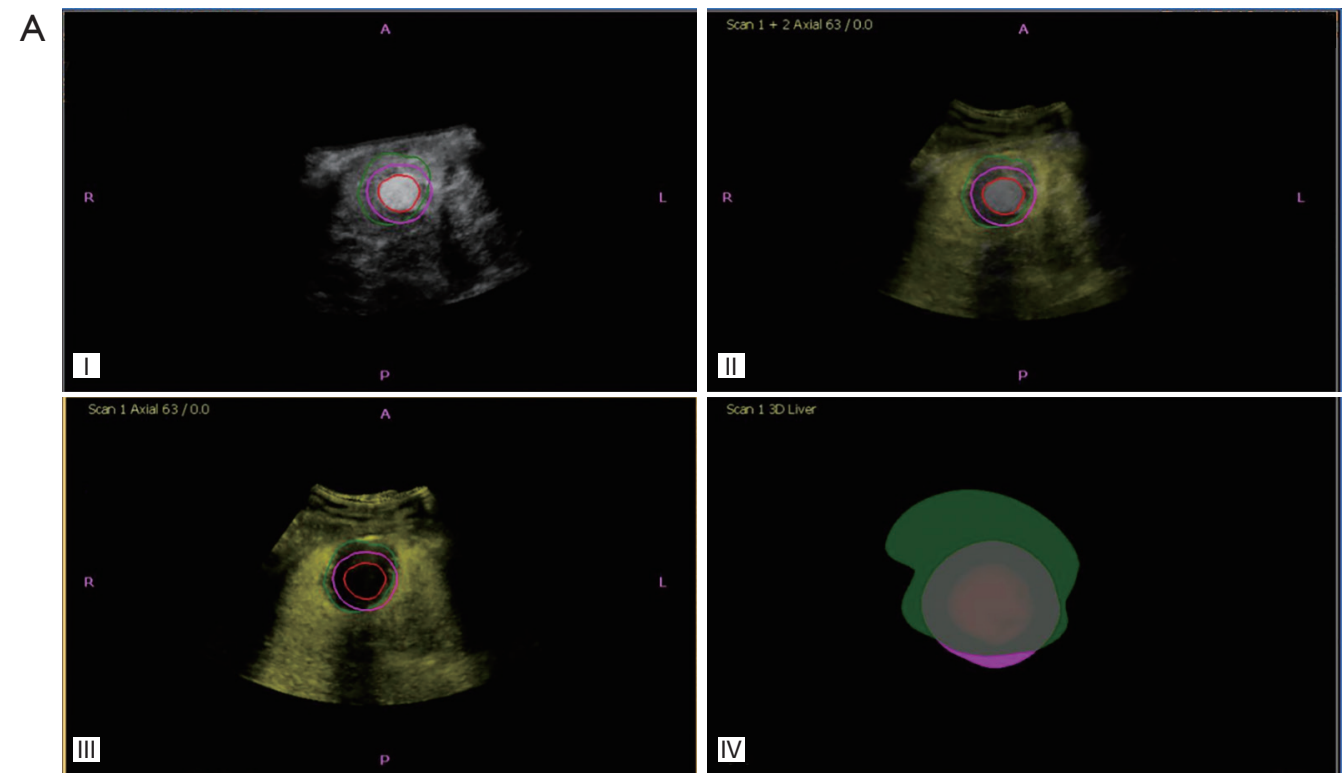

B
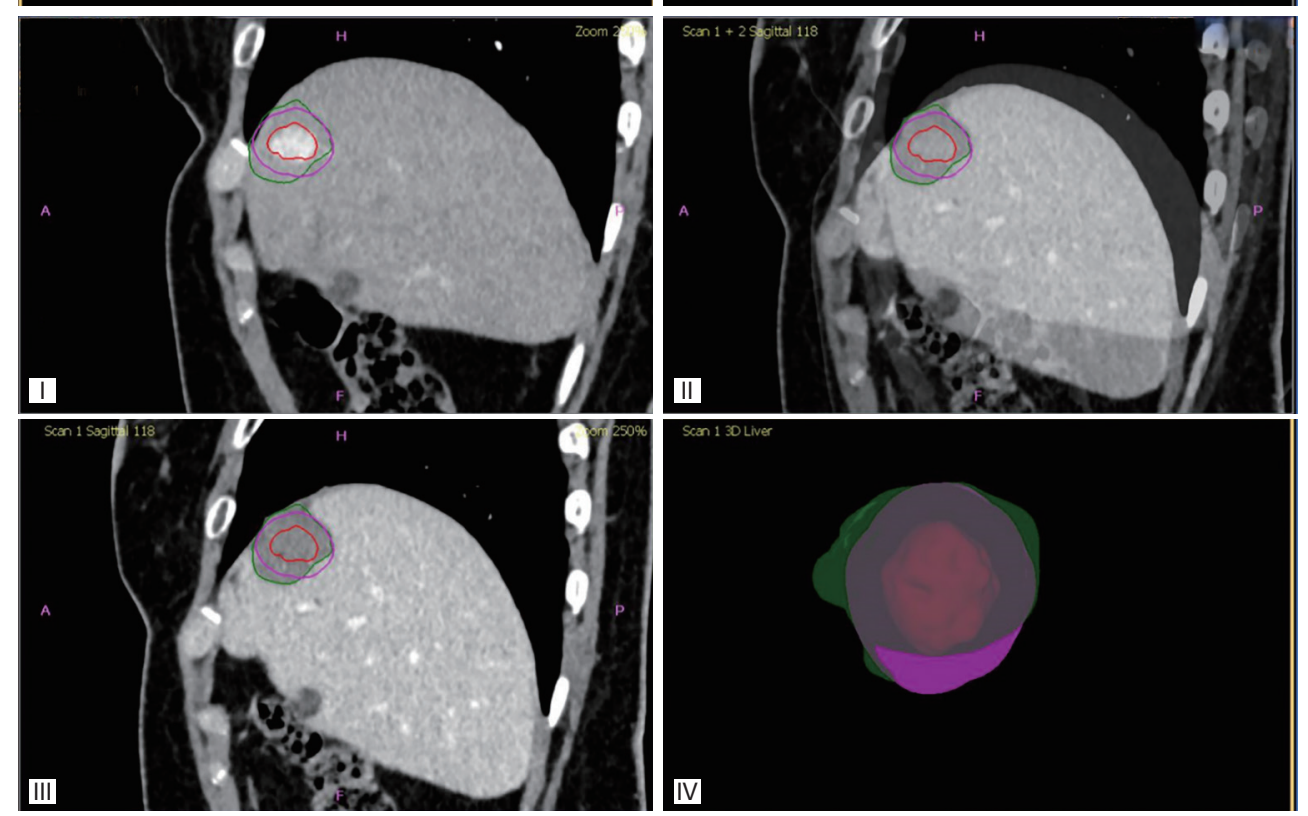

Figure 5 Images of a case with complete ablation of the tumor but not the safety margin. (A) Shows the 3D CEUS fusion result and (B) shows the CT fusion result. (I) shows the lesion before treatment; (II) shows the fusion of the pre- and post-treatment images; (III) shows the ablation area after treatment; and (IV) shows the 3D view of the lesion with the safety margin and the ablation area. 3D CEUS, threedimensional contrast-enhanced ultrasound.

completely ablated. Among them, the ablation margin of 14 tumors was $<5 \mathrm{~mm}$ (Figure 5), and the ablation margin of the other 9 tumors was $>5 \mathrm{~mm}$ (Figure 6).

For the 9 tumors with a safety margin $>5 \mathrm{~mm}$, the uncovered safety margin volume was $0 \mathrm{~mL}$, and the mean proportion of the uncovered safety margin to the total safety margin was 0 . For the 14 tumors with a safety margin $<5 \mathrm{~mm}$, the mean uncovered safety margin volume was $0.3-7.6$ $(2.27 \pm 2.11) \mathrm{mL}$, and the mean proportion of the uncovered safety margin to the total safety margin was $16.8 \%$.

\section{Enhanced CT fusion and evaluation of the ablation margin}

The success rate of enhanced CT fusion of images before 

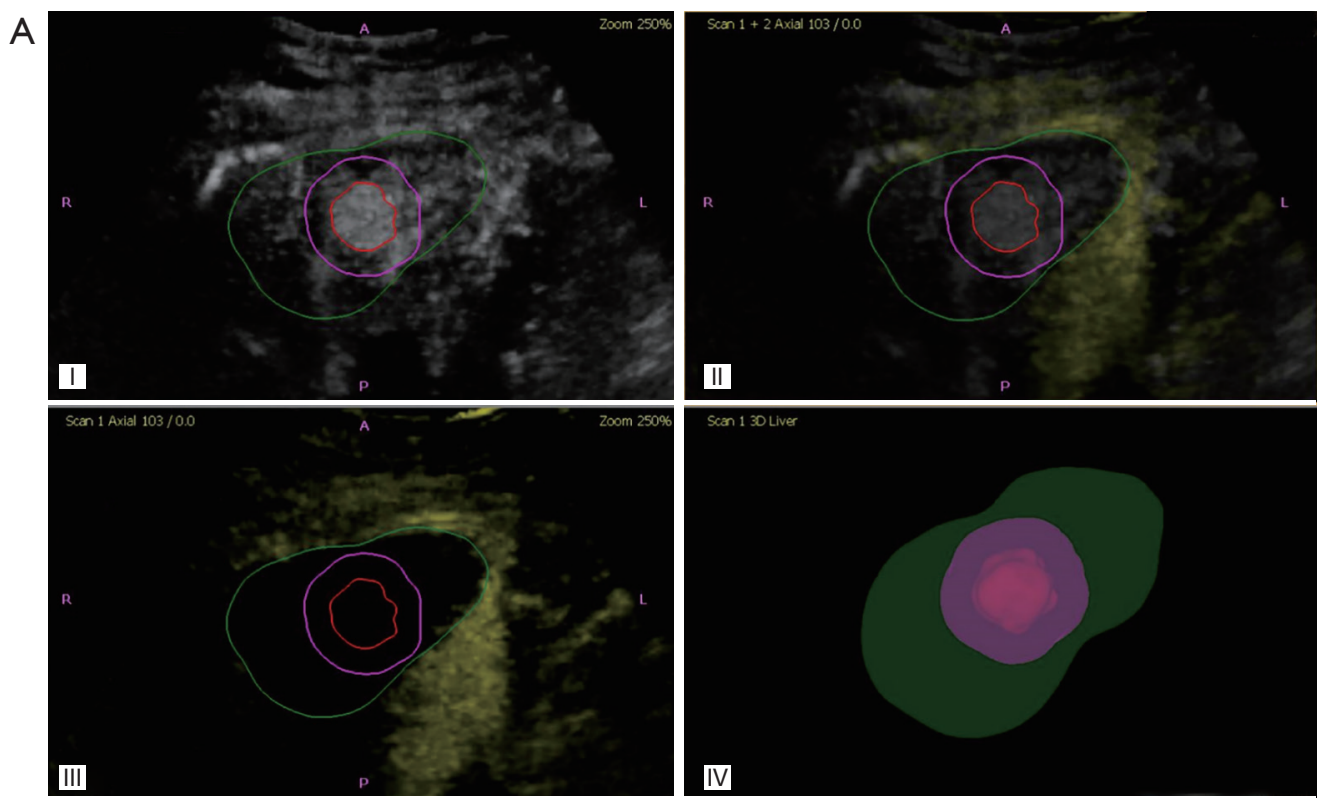

B
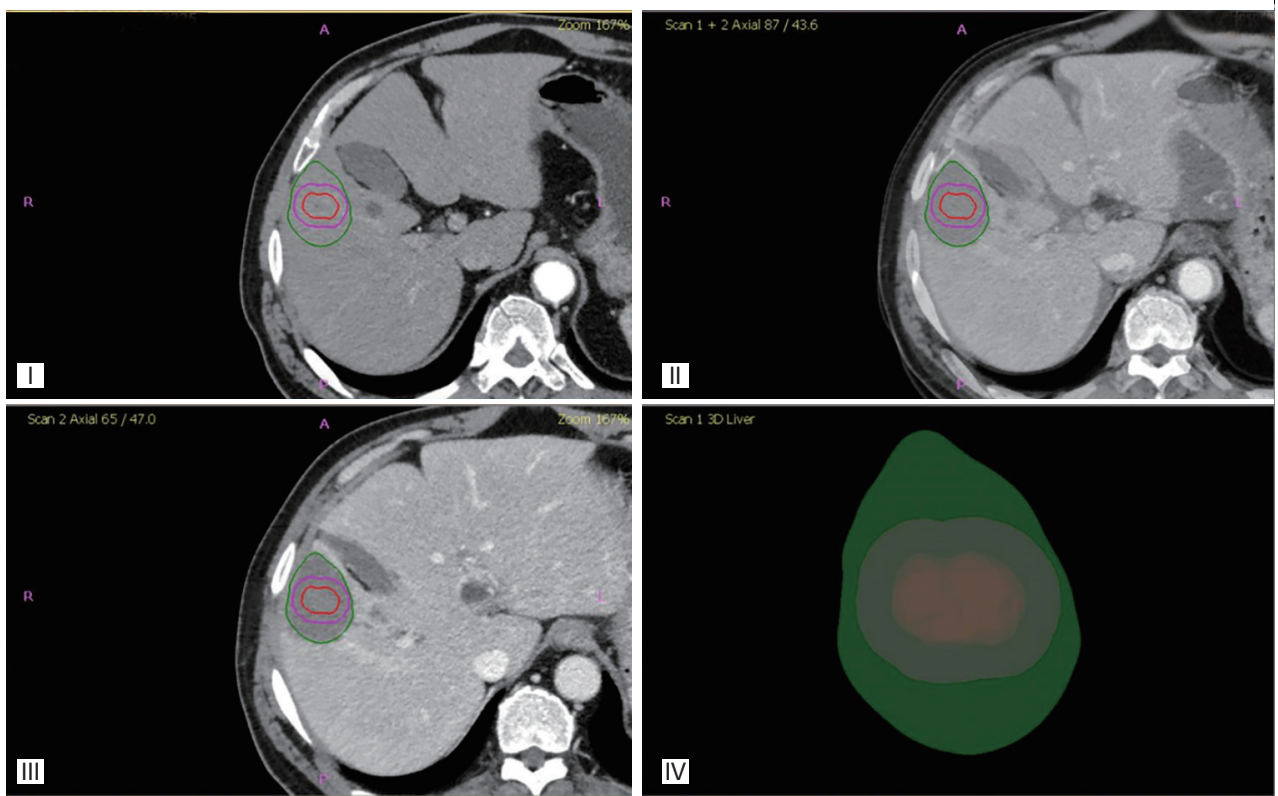

Figure 6 Images showing a case of complete ablation with safety margin. (A) Shows the 3D CEUS fusion result and (B) shows the CT fusion result. (I) shows the lesion before treatment; (II) shows the fusion of the pre- and post-treatment images; (III) shows the ablation area after treatment; and (IV) shows the 3D view of the lesion with the safety margin and ablation area. 3D CEUS, three-dimensional contrastenhanced ultrasound.

and after MWA treatment was 100\% (24/24), and the fusion time was $3-8(4.6 \pm 1.5)$ minutes. The 23 tumors evaluated by $3 \mathrm{D}$ CECT fusion were completely ablated (one lesion excluded due to the failure of 3D CEUS fusion imaging). The ablation margin of 13 tumors was $<5 \mathrm{~mm}$, and the ablation margin of 10 tumors was $>5 \mathrm{~mm}$.

\section{Comparison of ablation boundaries of 3D CEUS fusion and enhanced CT (Table 2)}

The 3D CEUS and enhanced CT fusion methods showed excellent consistency, and the Kappa value was 0.911 $(\mathrm{P}=0.000012)$. 
Table 2 Comparison of ablation boundaries of 3D CEUS fusion and enhanced CT

\begin{tabular}{lccc}
\hline \multirow{2}{*}{ 3D CEUS ablation margin } & \multicolumn{2}{c}{ CECT ablation margin } \\
\cline { 2 - 4 } & $<5 \mathrm{~mm}$ & $>5 \mathrm{~mm}$ \\
\hline$<5 \mathrm{~mm}$ & 13 & 1 \\
$>5 \mathrm{~mm}$ & 0 & 9 \\
\hline
\end{tabular}

3D CEUS, three-dimensional contrast-enhanced ultrasound.

\section{Discussion}

This study has presented a novel preoperative and postoperative 3D CEUS fusion imaging technique for the evaluation of safety margins of HCC immediately after thermal ablation. This fusion imaging technique based on the mono-modality imaging of CEUS is convenient and feasible for application in clinical practice using a semiautomatic $3 \mathrm{D}$ contouring method. The ablation result can be evaluated during the ablation process, and the ablation can be performed in time when the tumor is not completely ablated, to reduce the recurrence rate. Our results show that the performance of pre- and post-treatment $3 \mathrm{D}$ CEUS fusion imaging for evaluating the ablation margin is comparable with that of pre- and post-treatment CECT fusion imaging. Furthermore, the evaluation algorithm and image visualization are significantly improved with $3 \mathrm{D}$ CEUS fusion. Thus, the residual ablation volume to cover the safety margin and the proportion of the residual ablation volume to the total ablation volume can be calculated, which can assist physicians in making an appropriate therapeutic decision for the next step.

Thermal ablation, the third radical treatment method for early HCC, is widely used in clinical practice due to its minimally invasive nature. This treatment has similar longterm survival to liver resection; however, it has a higher tumor recurrence rate, especially local tumor recurrence, which may hinder the prognosis of patients (20). In the early period of thermal ablation, incomplete ablation was found to be the most common reason for local tumor recurrence. Consequently, some assisted methods were adopted to achieve complete ablation $(21,22)$. In recent years, many studies have confirmed that the ablation margin is closely related to local recurrence $(23,24)$. Thus, evaluation of the ablation margin becomes more and more important in the clinical treatment of HCC.

Fusion imaging has been confirmed to be a good method for evaluating safety margins. Preoperative and postoperative CT/MRI-CT/MRI and CT/MRI-
CEUS are the most widely used fusion imaging methods $(25,26)$. Multimodal fusion imaging has disadvantages of being inconvenient, expensive, and untimely. So monomodality fusion imaging methods, such as preoperative and postoperative 3D US-2D CEUS fusion imaging, have been presented to evaluate ablation margins $(13,26)$. The use of mono-modality fusion can also reduce problems caused by posture and breathing movement that lead to the failure of multi-modality fusion. Besides, mono-modality imaging is also convenient and efficient because it can display realtime images. Compared to 2D CEUS imaging, 3D CEUS provides more spatial structural information; therefore, we proposed its use to evaluate the results of thermal ablation in patients with HCC.

In this study, we verified the availability of using 3D CEUS fusion for the evaluation of ablation margin through clinical data. By using the simultaneouslyacquired 3D B-mode volume along with the 3D CEUS volume for registration, we were able to achieve a high fusion success rate, which illustrates the feasibility of this method. In our study, registration failed in only 1 case, due to poor respiratory coordination caused by severe pain from thermal ablation. When clinicians acquired the images from this patient, the image quality was poor, which resulted in the failure of fusion. In this study, we performed CT/MRI fusion within 1 week after ablation, which served as a reference standard. This was confirmed to be an effective method to reflect the real margin after ablation. Comparison of the 3D CEUS fusion results with those of CT fusion showed the 2 methods to have a high level of consistency. The kappa coefficient was statistically significant, suggesting 3D CEUS fusion is clinically feasible for ablation assessment.

Some previous studies also used preoperative and postoperative $3 \mathrm{D}$ CEUS to evaluate the ablation margin of HCC after thermal ablation $(17,27)$. Similar to our study, Ye et al. found that 3D CEUS fusion imaging enabled evaluation of the ablation margin of HCC immediately after radiofrequency ablation with an accuracy comparable to that 
of CT fusion imaging (17). Compared with previous study, the technique presented in our study has several advantages. Firstly, in our study, the real visual 3D technology was used to view 3D CEUS fusion images, whereas previous studies displayed 3D CEUS slice-by-slice (27). In our study, we significantly improved the visualization of fusion images by using a 3D view of the tumor volume, safety margin volume, and ablation margin volume with different translucent colors, which can display the tumor, safety margin, and ablation margins in a relatively straightforward way. Through this function, we were able to determine whether the lesion had been completely ablated, and whether the ablation area had reached the safety margin. Secondly, in previous studies $(17,27)$, the ablation margin was measured on the 3D CEUS fusion images based on the shortest distance between the tumor margin and the ablation area. In our study, once the ablation area had been segmented, the residual ablation volume needed to achieve safety margin and the proportion of the residual ablation volume to the whole ablation volume could be calculated automatically. Besides, the 3D CEUS fusion image and the corresponding 2D plane can also show the location of the residual ablation region, which can help the operator to find the appropriate needle path if necessary. Thirdly, to improve the accuracy of registration between the $3 \mathrm{D}$ US volumes, we used 3D US volume under B-Mode as a reference. With a higher mechanical index, the increased visibility could help the operator to find landmarks more easily. Furthermore, compared to previous studies using a matrix probe $(17,27)$, we obtained the $3 \mathrm{D}$ images using the free-arm method, which can reduce the influence of the ribs and is suitable for larger tumors.

The limitations of this study are as follows. First, it was single-center study with a small sample size and short follow-up time; thus, we could only determine the feasibility and accuracy of ablation using 3D CEUS fusion, but not the long term results. Also, for large tumors (diameter $>5 \mathrm{~cm}$ ), it was difficult to obtain a 3D US volume with full coverage of the tumor and especially the ablation areas.

In conclusion, 3D CEUS fusion has a high success rate and can be performed quickly. It also shows good consistency with enhanced CT/MRI fusion. Therefore, it is a feasible method for evaluating the immediate efficacy of thermal ablation in patients with HCC.

\section{Acknowledgments}

The authors thank Thomas TANG and Junping DENG who are working in Philips for their assistance in data collection, statistical analysis and interpretation.

Funding: This work was supported by Tianjin health and Health Committee (Grant No. MS20017, KJ20170).

\section{Footnote}

Reporting Checklist: The authors have completed the MDAR checklist. Available at http://dx.doi.org/10.21037/jgo-21-46

Data Sharing Statement: Available at http://dx.doi. org/10.21037/jgo-21-46

Conflicts of Interest: All authors have completed the ICMJE uniform disclosure form (available at http://dx.doi. org/10.21037/jgo-21-46). The authors have no conflicts of interest to declare.

Ethical Statement: The authors are accountable for all aspects of the work in ensuring that questions related to the accuracy or integrity of any part of the work are appropriately investigated and resolved. Approval for this prospective study was granted by the institutional review board of Tianjin Third Central Hospital (No. IRB2018017-01), and informed consent was obtained from all patients. All procedures performed in this study involving human participants were in accordance with the Declaration of Helsinki (as revised in 2013).

Open Access Statement: This is an Open Access article distributed in accordance with the Creative Commons Attribution-NonCommercial-NoDerivs 4.0 International License (CC BY-NC-ND 4.0), which permits the noncommercial replication and distribution of the article with the strict proviso that no changes or edits are made and the original work is properly cited (including links to both the formal publication through the relevant DOI and the license). See: https://creativecommons.org/licenses/by-nc-nd/4.0/.

\section{References}

1. Tombesi P, Di Vece F, Sartori S. Resection vs thermal ablation of small hepatocellular carcinoma: What's the first choice? World J Radiol 2013;5:1-4.

2. Bruix J, Sherman M. American Association for the Study of liver D. Management of hepatocellular carcinoma: an update Hepatology 2011;53:1020-2.

3. Shiina S, Sato K, Tateishi R, et al. Percutaneous ablation 
for hepatocellular carcinoma: comparison of various ablation techniques and surgery. Can J Gastroenterol Hepatol 2018;2018:4756147.

4. Shi J, Sun Q, Wang Y, et al. Comparison of microwave ablation and surgical resection for treatment of hepatocellular carcinomas conforming to $\mathrm{M}$ ilan criteria. J Gastroenterol Hepatol 2014;29:1500-7.

5. Kang TW, Lim HK, Lee MW, et al. Perivascular versus nonperivascular small HCC treated with percutaneous RF ablation: retrospective comparison of long-term therapeutic outcomes. Radiology 2014;270:888-99.

6. Velez E, Goldberg SN, Kumar G, et al. Hepatic thermal ablation: effect of device and heating parameters on local tissue reactions and distant tumor growth. Radiology 2016;281:782-92.

7. Okuwaki Y, Nakazawa T, Shibuya A, et al. Intrahepatic distant recurrence after radiofrequency ablation for a single small hepatocellular carcinoma: risk factors and patterns. J Gastroenterol 2008;43:71-8.

8. Liu M, Huang G-1, Xu M, et al. Percutaneous thermal ablation for the treatment of colorectal liver metastases and hepatocellular carcinoma: a comparison of local therapeutic efficacy. Int J Hyperthermia 2017;33:446-53.

9. Ding J, Jing X, Wang Y, et al. Thermal ablation for hepatocellular carcinoma: a large-scale analysis of longterm outcome and prognostic factors. Clin Radiol 2016;71:1270-6.

10. Calandri M, Mauri G, Yevich S, et al. Fusion imaging and virtual navigation to guide percutaneous thermal ablation of hepatocellular carcinoma: a review of the literature. Cardiovasc Intervent Radiol 2019;42:639-47.

11. Jiang C, Liu B, Chen S, et al. Safety margin after radiofrequency ablation of hepatocellular carcinoma: precise assessment with a three-dimensional reconstruction technique using CT imaging. Int J Hyperthermia 2018;34:1135-41.

12. Wang X-L, Li K, Su Z-Z, et al. Assessment of radiofrequency ablation margin by MRI-MRI image fusion in hepatocellular carcinoma. World J Gastroenterol 2015;21:5345.

13. Minami Y, Minami T, Chishina H, et al. US-US fusion imaging in radiofrequency ablation for liver metastases. Dig Dis 2016;34:687-91.

14. Xu EJ, Lv SM, Li K, et al. Immediate evaluation and guidance of liver cancer thermal ablation by threedimensional ultrasound/contrast-enhanced ultrasound fusion imaging. Int J Hyperthermia 2018;34:870-6.

15. Minami Y, Minami T, Hagiwara S, et al. Ultrasound- ultrasound image overlay fusion improves real-time control of radiofrequency ablation margin in the treatment of hepatocellular carcinoma. Eur Radiol 2018;28:1986-93.

16. Wang Y, Jing X, Ding J. Clinical value of dynamic 3-dimensional contrast-enhanced ultrasound imaging for the assessment of hepatocellular carcinoma ablation. Clin Imaging 2016;40:402-6.

17. Ye J, Huang G, Zhang X, et al. Three-dimensional contrast-enhanced ultrasound fusion imaging predicts local tumor progression by evaluating ablative margin of radiofrequency ablation for hepatocellular carcinoma: a preliminary report. Int J Hyperthermia 2019;36:55-64.

18. Heimbach JK, Kulik LM, Finn RS, et al. AASLD guidelines for the treatment of hepatocellular carcinoma. Hepatology 2018;67:358-80.

19. Ding J, Long L, Zhang X, et al. Contrast-enhanced ultrasound LI-RADS 2017: comparison with CT/MRI LIRADS. Eur Radiol 2021;31:847-54.

20. Yu C, Wu S, Zhao J, et al. Evaluation of efficacy, safety and treatment-related outcomes of percutaneous radiofrequency ablation versus partial hepatectomy for small primary liver cancer meeting the Milan criteria: A systematic review and meta-analysis of randomized controlled trials. Clin Res Hepatol Gastroenterol 2020;44:718-32.

21. Choe WH, Kim YJ, Park HS, et al. Short-term interval combined chemoembolization and radiofrequency ablation for hepatocellular carcinoma. World J Gastroenterol 2014;20:12588.

22. Shimose $S$, Tanaka $M$, Iwamoto $H$, et al. Prognostic impact of transcatheter arterial chemoembolization (TACE) combined with radiofrequency ablation in patients with unresectable hepatocellular carcinoma: Comparison with TACE alone using decision-tree analysis after propensity score matching. Hepatol Res 2019;49:919-28.

23. Ju J-X, Zeng Q-J, Xu E-J, et al. Intraprocedural contrastenhanced ultrasound-CT/MR fusion imaging assessment in HCC thermal ablation to reduce local tumor progression: compared with routine contrast-enhanced ultrasound. Int J Hyperthermia 2019;36:785-93.

24. An C, Li X, Liang P, et al. A tumor map generated from three-dimensional visualization of image fusion for the assessment of microwave ablation of hepatocellular carcinoma: a preliminary study. Cancer Manag Res 2019;11:1569.

25. Xu E, Li K, Long Y, et al. Intra-procedural CT/MRultrasound fusion imaging helps to improve outcomes of thermal ablation for hepatocellular carcinoma: results in 
502 nodules. Ultraschall Med 2019. [Epub ahead of print]. doi: 10.1055/a-1021-1616.

26. Xu E, Long Y, Li K, et al. Comparison of CT/MRICEUS and US-CEUS fusion imaging techniques in the assessment of the thermal ablation of liver tumors. Int J Hyperthermia 2019;35:159-67.

27. Zhang X, Huang G, Ye J, et al. 3-D Contrast-Enhanced

Cite this article as: Ding J, Wang D, Zhou Y, Zhao L, Zhou H, Jing X, Wang Y. A novel mono-modality fusion imaging method based on three-dimensional contrast-enhanced ultrasound for the evaluation of ablation margins after microwave ablation of hepatocellular carcinoma. J Gastrointest Oncol 2021;12(1):184195. doi: 10.21037/jgo-21-46
Ultrasound Fusion Imaging: A New Technique to Evaluate the Ablative Margin of Radiofrequency Ablation for Hepatocellular Carcinoma. Ultrasound Med Biol 2019;45:1933-43.

(English Language Editor: J. Reynolds) 